Pacific Journal of Mathematics

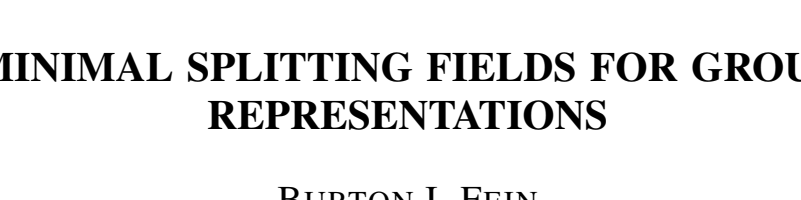




\title{
MINIMAL SPLITTING FIELDS FOR GROUP REPRESENTATIONS
}

\author{
BuRton FeIN
}

Let $T$ be a complex irreducible representation of a finite group $G$ of order $n$ and let $\chi$ be the character afforded by $T$. An algebraic number field $K \supset Q(\chi)$ is a splitting field for $\chi$ if $T$ can be written in $K$. The minimum of $[K: Q(\chi)]$, taken over all splitting fields $K$ of $\chi$, is the Schur index $m_{Q}(\chi)$ of $\chi$. In view of the famous theorem of $\mathbf{R}$. Brauer that $Q\left(e^{2 \pi i} / n\right)$ is a splitting field for $\chi$, it is natural to ask whether there exists a splitting field $L$ with $Q\left(e^{2 \pi i} / n\right) \supset L \supset Q(\chi)$ and $[L: Q(\chi)]=$ $m_{Q}(\chi)$. In this paper examples are constructed which show that such a splitting field $L$ does not always exist. Sufficient conditions are also obtained which guarantee the existence of a splitting field $L$ as above.

Throughout this paper $Q$ will denote the field of rational numbers. If $K$ is an algebraic number field and $p$ is a prime of $K$, we denote the completion of $K$ at $p$ by $K_{p}$. If $A$ is a simple component of a group algebra over $Q$, the center of $A$ being $K$, and $\pi_{1}$ and $\pi_{2}$ are primes of $K$ extending the rational prime $p$, then the indices of $A \otimes_{K} K_{\pi_{1}}$ and $A \otimes_{K} K_{\pi_{2}}$ are equal [2, Theorem 1]. We write $l . i_{{ }_{p}} A$ for this common value and refer to $l_{.} i_{{ }_{p}} A$ as the $p$-local index of $A$. If $L \supset K$ and $L$ is an abelian extension of $Q$, we refer to the ramification degree of a prime $\pi$ of $K$ from $K$ to $L$ as the $q$-ramification degree where $\pi$ extends the rational prime $q$. Clearly, this does not depend on the choice of $\pi$. We use similar notation when referring to residue class degrees.

Throughout this paper $\chi$ will denote an irreducible complex character of a finite group $G$ of order $n$. There is a unique constituent $\mathscr{A}$ of the group algebra of $G$ over $Q(\chi)$ corresponding to $\chi$ in the sense that the representation of $G$ afforded by a minimal left ideal of $\mathscr{A}$ is equivalent to $m_{Q}(\chi) T$, where $T$ affords $\chi$. If $D$ is the division algebra component of $\mathscr{A}$ we say that $D$ (and $\mathscr{A}$ ) is associated with $\chi$. The index of $D$ equals $m_{Q}(\chi)$ and $\chi$ is realizable in $K$ if and only if $K$ is a splitting field for $D$. We refer the reader to [1] for the relevant theory of algebras assumed.

We denote a primitive $m$ th root of unity by $\varepsilon_{m}$. Gal $(L / K)$ denotes the Galois group of $L$ over $K$, and [ $L: K]$ the degree of $L$ over $K$. If $A$ and $B$ are two central simple $K$-algebras we write $A \sim B$ to denote that $A$ and $B$ are similar in the Brauer group of $K$. 631]:

A special case of the following lemma is proved in [6, page 
LEMMA. Let $F$ be the completion of an algebraic number field at a finite prime and suppose the residue class field of $F$ has $q$ elements. Let $p$ be a prime, $p \nmid q$, and suppose $p^{t} \mid q-1, p^{t+1} \nmid q-1$. Let $E$ be a cyclic extension of $F$ of degree $p^{e} \cdot p^{f}$ where $p^{e}, e>0$, is the ramification degree of $E$ over $F$. Let $\langle\sigma\rangle=\operatorname{Gal}(E / F)$ and let $\varepsilon_{p^{s}} \in F$. We have:

(1) Let $p^{t}=2$ so $\varepsilon_{p^{s}}=-1$. Then the cyclic algebra $(E, \sigma,-1)$ has index 2.

(2) Suppose $p^{t} \geqq 3$ and $s \geqq v>0$. Then $\left(E, \sigma, \varepsilon_{p^{s}}\right)$ has index $p^{v}$ if and only if $t=e+s-v$.

Proof. By Hensel's lemma, $\varepsilon_{p^{t}} \in F, \varepsilon_{p^{t+1}} \notin F$. Let $[K: F]=p^{f}, K$ unramified over $F$. All $p$-power roots of unity in $E$ are in $K$. If $p^{t} \geqq 3$, an easy induction shows that $E$ contains a primitive $p^{t^{+} f}$ th root of unity but does not contain a primitive $p^{t+f+1}$ th root of unity. If $p^{t}=2$ and $f>0$, then $E$ contains a primitive $2^{2+f}$ th root of unity but not a primitive $2^{3+f}$ th root of unity. If $p^{t}=2$ and $f=0$, then $E$ does not contain $\varepsilon_{4}$. From the theory of cyclic algebras over local fields, $\left(E, \sigma, \varepsilon_{p^{s}}\right)$ has index $p^{v}$ if and only if $\varepsilon_{p^{s-v}}$ is a norm from $E$ to $F$ but $\varepsilon_{p^{s-v+1}}$ is not a norm. Suppose $\varepsilon_{p^{s-v}}$ is a norm from $E$ to $F$. Let $N$ denote the norm map from $E$ to $F$. Since $\varepsilon_{p^{s-v}}$ is a unit, $\varepsilon_{p^{s-v}}=N(\gamma)$ where $\gamma$ is a unit of $E$. Let $U_{E}, U_{E^{1}}$ denote, respectively, the units and the units $(\bmod 1)$ of $E$. We have $U_{E / U_{E}{ }^{1}} \cong \bar{E}^{*}$, the multiplicative group of the residue claas field of $E$. Since $E$ and $K$ have the same residue class field, there is a root of unity $\delta$ in $K$ with $\gamma U_{E^{1}}=\delta U_{E^{1}}$. Since $N(\delta) U_{F^{1}}=\varepsilon_{p^{s-v}} U_{F^{1}}=N(\delta) U_{F^{1}}$, we may assume that $\delta$ has $p$-power order. Let $N^{\prime}$ denote the norm from $K$ to $F$. Then $N(\delta)=N^{\prime}\left(\delta^{p^{e}}\right)$ since $\delta \in K$. Since Gal $(K / F)$ is generated by the Frobenius automorphism, we have $N(\delta)=\delta^{m p^{e}}$ where

$$
m=\left(q^{p f}-1\right) /(q-1) .
$$

Suppose (1) holds so $p^{t}=2, \varepsilon_{p^{s}}=-1 .(E, \sigma,-1)$ has index 1 or 2 and we have index 1 if and only if -1 is a norm from $E$. By the argument above, if -1 is a norm, then $-1 U_{F^{1}}=\delta^{m 2^{e}} U_{F^{1}}$ where $\delta$ is a 2-power root of unity, $e>0$, and $m=\left(q^{2 f}-1\right) /(q-1)$. One verifies easily that $\delta^{m 2^{e}}=1$, a contradiction.

Now suppose (2) holds. Assuming $\varepsilon_{p^{s-v}}$ is a norm from $E$ we obtain, as above, that $N(\delta)$ is a power of a primitive $p^{t-e}$ th root of unity. Thus $t-e \geqq s-v$ so $t \geqq s+e-v$. Conversely, if $t=s+$ $e-v$, then $E$ contains a primitive $p^{s+e+f-v}$ th root of unity $\zeta$. An easy calculation using the Frobenius automorphism shows that $N\left(\zeta^{u}\right)=$ $\varepsilon_{p^{s-v}}$ for some $u$. Let $\mathscr{A}=\left(E, \sigma, \varepsilon_{p^{s}}\right)$ so $\mathscr{A}^{p^{v}} \sim\left(E, \sigma, \varepsilon_{p^{s-v}}\right)$. If $t=s+e-v$, then we have shown that $\mathscr{A}^{p^{v}} \sim F$. If $\mathscr{A}^{p^{v-1}} \sim F$, 
then we would have $\mathrm{t} \geqq s+e-v+1$ which is not the case. Thus $t=s+e-v$ implies $\mathscr{A}$ has index $p^{v}$. Conversely, if $\mathscr{A}$ has index $p^{v}$, then $t \geqq s+e-v$. If $t \geqq s+e-v+1$ we would have $\mathscr{A}^{p^{v-1}} \sim$ $F$. Thus $t=s+e-v$, proving the lemma.

We can now construct an example (actually one for each prime $p$ ) of an irreducible character $\chi$ of a finite group $G$ of order $n$ such that $m_{Q}(\chi)=p$ but no subfield $L$ of $Q\left(\varepsilon_{n}\right)$ with $[L: Q(\chi)]=p$ is a splitting field for $\chi$.

EXAMPLE. Let $p$ be an arbitrary prime. Let $r$ be prime, $r \equiv 1\left(\bmod p^{2}\right), r \neq \equiv 1\left(\bmod p^{3}\right)$. Let $q$ be a prime, $q \equiv 1(\bmod r), q \equiv$ $1\left(\bmod p^{4}\right)$, and $q \not \equiv 1\left(\bmod p^{5}\right)$. Let $F$ be the subfield of $Q\left(\varepsilon_{q}\right)$ with $\left[Q\left(\varepsilon_{q}\right): F\right]=p^{4}$ and let $E$ be the subfield of $Q\left(\varepsilon_{r}\right)$ with $\left[Q\left(\varepsilon_{r}\right): E\right]=p^{2}$. Let $\langle\sigma\rangle=\operatorname{Gal}\left(Q\left(\varepsilon_{p^{3} q r}\right) / F\left(\varepsilon_{p^{3} r}\right)\right)$ and $\langle\tau\rangle=\operatorname{Gal}\left(Q\left(\varepsilon_{p^{3} q r}\right) / E\left(\varepsilon_{p^{3} q}\right)\right)$. Let $K$ be the fixed field of $\langle\sigma \tau\rangle$. Then $K\left(\varepsilon_{q}\right)=Q\left(\varepsilon_{p^{3} q r}\right)$ and $\left[K\left(\varepsilon_{q}\right): K\right]=p^{4}$. Since $q$ is totally ramified from $E F\left(\varepsilon_{p^{3}}\right)$ to $F\left(\varepsilon_{p^{3} q}\right)$ and splits completely from $E F\left(\varepsilon_{p^{3}}\right)$ to $E\left(\varepsilon_{p^{3} r}\right)$, we see that $q$ is totally ramified from $E F\left(\varepsilon_{p^{3}}\right)$ to $K$. Thus the ramification degree of $q$ from $K$ to $K\left(\varepsilon_{q}\right)$ is $p^{2}$ and the residue class degree is 1 .

Let $G=\langle w, x, y, z| w^{q}=x^{r}=z^{p^{3}}=1, y^{p^{4}}=z, z$ central, $(w, x)=1$, $\left.y^{-1} w y=w^{a}, y^{-1} x y=x^{b}\right\rangle$ where $\sigma \tau\left(\varepsilon_{q}\right)=\left(\varepsilon_{q}\right)^{a}$ and $\sigma \tau\left(\varepsilon_{r}\right)=\left(\varepsilon_{r}\right)^{b}$. The cyclic algebra $\mathscr{A}=\left(Q\left(\varepsilon_{p^{3} q r}\right), \sigma \tau, \varepsilon_{p^{3}}\right)$ is a homomorphic image of the group algebra of $G$ over $Q$ and so there exists a complex irreducible representation $T$ of $G$ with character $\chi$ such that the enveloping algebra of $T$ is $\mathscr{A}$ and $Q(\chi)=K$. The index of $\mathscr{A}$ equals $m_{Q}(\chi)$.

By the lemma we see that $\mathscr{A}$ has $q$-local index $p$. Since $K\left(\varepsilon_{q}\right)=Q\left(\varepsilon_{p^{3} q r}\right), r$ is unramified from $K$ to $Q\left(\varepsilon_{p^{3} q r}\right)$ and so the $r$-local index of $\mathscr{A}$ is 1 . Since the 2-local index is at most 2 [7, Satz 11] and at infinite primes $\mathscr{A}$ can only have index 1 or 2 , we conclude that $m_{Q}(\chi)=p . \quad|G|=p^{7} q r$ and $\operatorname{Gal}\left(Q\left(\varepsilon_{p^{7} q r}\right) / K\right) \cong C_{p^{4}} \times C_{p^{4}}$. Since $q \equiv 1\left(\bmod p^{4}\right)$ we see that $q$ splits completely in the unique extension $J$ of $K, J \subset Q\left(\varepsilon_{p^{7} q r}\right)$, Gal $(J / K)=C_{p} \times C_{p}$. It follows, therefore, that $q$ splits completely in every subfield of $Q\left(\varepsilon_{p^{7} q r}\right)$ of degree $p$ over $K$ and so $T$ is not realizable in any subfield $L$ of the $|G|$ th roots of unity with $[L: Q(\chi)]=p$.

We next prove that under certain conditions there always exists a subfield $L$ of the order of $|G|$ th roots of unity which is a splitting field for $\chi$ and where $[L: Q(\chi)]=m_{Q}(\chi)$.

THEOREM. Let $\chi$ be a complex irreducible character of a finite group $G$ of exponent $n$ with $m_{Q}(\chi) \geqq 3$. Assume either (a) or (b) below hold:

(a) $Q(\chi)=Q\left(\varepsilon_{m}\right)$ for some $m$.

(b) $n=p^{a} q^{b}$ where $p$ and $q$ are primes, $p<q$. 
Then there exists a subfield $L$ of $Q\left(\varepsilon_{n}\right)$ with $[L: Q(\chi)]=m_{Q}(\chi)$ and such that $L$ is a splitting field for $\chi$.

Proof. By a standard reduction using the Brauer-Witt theorem $[8, \S 2]$, we may assume that $m_{Q}(\chi)$ is a prime power. Since if (b) holds, $m_{Q}(\chi)$ is a power of $p$ by [7, Satz 10], we will assume that $m_{Q}(\chi)=p^{c}$.

Let $K$ be the subfield of $Q\left(\varepsilon_{n}\right)$ such that $K \supset Q(\chi), p \nmid[K: Q(\chi)]$, and $\left[Q\left(\varepsilon_{n}\right): K\right]$ is a power of $p$. Let $D$ be the $Q(\chi)$-central division algebra associated with $\chi$. By the Brauer-Witt theorem [8, §2], $D \otimes_{Q(X)} K$ is similar to a crossed product $(K(\psi) / K, \beta)$ where $\psi$ is a linear character of a subgroup of $G, \beta$ is a factor set whose values are roots of unity, and where $\operatorname{Gal}(K(\psi) / K)$ is isomorphic to a factor group of a Sylow $p$-subgroup of $G$.

$Q(\chi)$ contains a primitive $m_{Q}(\chi)$ th root of unity [3, Theorem 1]. Since $m_{Q}(\chi) \geqq 3, Q(\chi)$ and $K$ are both totally imaginary. Thus the nonzero invariants of $D$ are at finite primes.

Suppose (a) holds, so $Q(\chi)=Q\left(\varepsilon_{m}\right)$. We may assume $m$ is not twice an odd number. We have $m_{Q}(\chi) \mid m$. If $r$ is a prime divisor of $m, r \neq p$, then since, for some $d,\left[Q\left(\varepsilon_{n}\right): K\right]=p^{d}, r$ is unramified from $K$ to $K(\psi)$. This implies that the $r$-local index of $D$ equals 1 . Now let $q_{1}, \cdots, q_{t}$ be the rational primes at which $D$ has nontrivial local index. Let the $q_{i}$-local index of $D$ be $p^{c_{i}}$. Then $c_{i} \leqq c$ for all $i$ and $c_{i}=c$ for some $i$ since $D$ has index $p^{c}$. Suppose $q_{i}$ is odd. By [7, Satz 10] $p^{c_{i}} \mid q_{i}-1$ and so $Q\left(\varepsilon_{q_{i}}\right)$ has a subfield $E_{i}$ with $\left[E_{i}\right.$ : $Q]=p^{c_{i}}$. Since $q_{i} \nmid m,\left[E_{i} Q(\chi): Q(\chi)\right]=p^{c_{i}}$ and $q_{i}$ is totally ramified from $Q(\chi)$ to $E_{i} Q(\chi)$. Let $L_{i}=E_{i} Q(\chi)$. By [3, Theorem 1], $\varepsilon_{p c_{i}} \in Q(\chi)$ and so $L_{i}=Q(\chi)\left(\alpha_{i}\right)$ where $\alpha_{i}^{p^{c} i} \in Q(\chi)$. If all of the $q_{2}$ are odd, let $\alpha=\alpha_{1} \alpha_{2} \cdots \alpha_{t}$. If $q_{1}=2$, say, let $a=\sqrt{-1} \alpha_{2} \cdots \alpha_{t}$. We note that $q_{1}$ can equal 2 only if $p^{c_{1}}=2$ and $\sqrt{-1} \notin Q(\chi)$ [7, Satz 11]. If this happens, then $4 \mid n$ by [4]. Thus $\alpha \in Q\left(\varepsilon_{n}\right)$. Since $\alpha^{p^{c}} \in Q(\chi)$, $[Q(\chi)(\alpha): Q(\chi)] \leqq p^{c}$. Since $q_{\imath}$ is ramified of degree $p^{c_{i}}$ from $Q(\chi)$ to $Q(\chi)(\alpha),[Q(\chi)(\alpha): Q(\chi)]=p^{c}$ and $Q(\chi)(\alpha)$ splits $D$. Thus $Q(\chi)(\alpha)$ is our desired field.

Assume (b) holds. $K(\psi)$ is an abelian extension of $K$ generated by roots of unity. Since $(K(\psi) / K, \beta)$ has index $p^{c}>1,(K(\psi) / K, \beta)$ has $q$-local index $p^{c}$ and so $q$ is ramified from $K$ to $K(\psi)$. This implies that $K(\psi) \supset K\left(\varepsilon_{q}\right)=K\left(\varepsilon_{q b}\right)$. Since $m_{Q}(\chi)=p^{c} \geqq 3$, if $p=2$ we see that $\sqrt{-1} \in K$. In view of [7, Satz 12] this implies that $q$ is the only prime of $Q$ with the $q$-local index of $(K(\psi) / K, \beta)$ different from 1 .

Let $\varepsilon_{p^{v}} \in K(\psi), \varepsilon_{p^{v+1}} \notin K(\psi)$. We note that $K(\psi)=Q\left(\varepsilon_{p^{v} q^{b}}\right)$ since $\left[Q\left(\varepsilon_{p^{a} q^{b}}\right): K\right]$ is a power of $p$. Let $\langle\sigma\rangle=\operatorname{Gal}\left(Q\left(\varepsilon_{p^{v} q^{b}}\right) / Q\left(\varepsilon_{p^{v}}\right)\right),\langle\tau\rangle=$ $\operatorname{Gal}\left(Q\left(\varepsilon_{p^{v} q^{b}}\right) / Q\left(\varepsilon_{q^{b}}\right)\right)$. Then $\left\langle\sigma^{i} \tau^{j}\right\rangle=\operatorname{Gal}\left(Q\left(\varepsilon_{p^{v} q^{b}}\right) / K\right)$ for some $i$ and $j$. Let $F_{1}$ and $F_{2}$ be, respectively, the fixed fields of $\left\langle\sigma^{i}\right\rangle$ and $\left\langle\tau^{j}\right\rangle$. Let 
$p^{e}$ and $p^{t}$ be, respectively, the order, of $\left\langle\sigma^{i}\right\rangle$ and $\left\langle\tau^{j}\right\rangle$. Let $L_{1}$ and $L_{2}$ be, respectively, the subfields of index $p^{e}$ and $p^{t}$ in $Q\left(\varepsilon_{q^{b}}\right)$ and $Q\left(\varepsilon_{p^{v}}\right)$. Then $F_{1}=L_{1}\left(\varepsilon_{p^{v}}\right)$ and $F_{2}=L_{2}\left(\varepsilon_{q^{b}}\right)$ and $F_{1} \cap F_{2}=L_{1} L_{2}$. Since $q$ is totally ramified from $L_{1} L_{2}$ to $F_{2}$ and is unramified from $L_{1} L_{2}$ to $F_{1}, q$ is totally ramified from $L_{1} L_{2}$ to $K$. Thus $e>t$ and $q$ has ramification degree $p^{e-t}$ from $K$ to $K(\psi)$.

Suppose $\left[K\left(\varepsilon_{p^{v}}\right): K\right]=p^{s}$. Then $\left(\sigma^{i} \tau^{j}\right)^{p^{s}}$ fixes $K\left(\varepsilon_{p^{v}}\right)$. Since $\sigma$ fixes $\varepsilon_{p^{v}}, \tau^{j p^{s}}$ fixes $\varepsilon_{p^{v}}$ and so $\tau^{j p^{s}}=1$. Thus $s \geqq t$. But $q$ is unramified from $K$ to $K\left(\varepsilon_{p v}\right)$ and so the ramification degree of $q$ from $K$ to $K(\psi)$ is at most $p^{e-s}$. Thus $e-s \geqq e-t$ so $s=t$. This shows that $q$ is totally ramified from $K\left(\varepsilon_{p^{v}}\right)$ to $K(\psi)$. Since $q$ is unramified from $K(\psi)$ to $K\left(\varepsilon_{p^{a} q} b\right)=Q\left(\varepsilon_{p^{a} q} b\right)$, we see that $K\left(\varepsilon_{p^{a}}\right)$ is the maximal extension of $K$ inside $Q\left(\varepsilon_{p^{a} q^{b}}\right)$ in which $q$ is unramified.

$Q\left(\varepsilon_{p^{a} q^{b}}\right)$ is not a cyclic extension of $K$ by [5]. Thus Gal $\left(Q\left(\varepsilon_{p^{a} q^{b}}\right) / K\right)$ is the direct product of two cyclic groups. Let $M_{1}$ and $M_{2}$ be subfields of $Q\left(\varepsilon_{p^{a} q^{b}}\right)$ such that $M_{1} \cap M_{2}=K, Q\left(\varepsilon_{p^{a} q^{b}}\right)=M_{1} M_{2}$, and $M_{1}$ and $M_{2}$ are cyclic extensions of $K$. Since $K\left(\varepsilon_{p a}\right)$ is cyclic over $K, q$ must be totally ramified in either $M_{1}$ or $M_{2}$. Suppose $q$ is totally ramified in $M_{1}$. By [5], since $Q\left(\varepsilon_{p^{a} q^{b}}\right)$ is cyclic over $M_{1}, M_{1}$ is a splitting field for $\chi$. Thus $M_{1}$ splits $(K(\psi) / K, \beta)$ and so $\left[M_{1}: K\right] \geqq p^{c}$. The subfield $L$ of $M_{1}$ with $[L: Q(\chi)]=p^{c}$ is the desired splitting field for $\chi$. This completes the proof of the theorem.

\section{REFERENCES}

1. A. A. Albert, Structure of algebras, Amer. Math. Soc. Colloq. Publ., 24, Providence, R. I., 1939.

2. M. Benard, The Schur subgroup, I, J. Algebra, 22 (1972), 374-377.

3. M. Benard and M. Schacher, The Schur subgroup II, J. Algebra, 22 (1972), 378-385.

4. B. Fein and T. Yamada, The Schur index and the order and exponent of a finite group, J. Algebra, 28 (1974), 496-498.

5. D. Goldschmidt and I. M. Isaacs, to appear, in J. Algebra.

6. M. Schacher, Cyclotomic splitting field, Proc. Amer. Math. Soc., 25 (1970), 630-633.

7. E. Witt, Die algebraische Struktur des Grupperringes einer endlicher Gruppe über einem Zahlkörper, J. Reine Angew, Math., 190 (1952), 231-245.

8. T. Yamada, Characterization of the simple components of the group algebras over the p-adic number field, J. Math. Soc. Japan, 23 (1971), 295-310.

Received October 15, 1973. This work was done under the sponsorships of NSF Grant GP-29068. AMS classification: 20C15

Oregon State University 



\section{PACIFIC JOURNAL OF MATHEMATICS}

\section{EDITORS}

RICHARD ARENS (Managing Editor)

University of California

Los Angeles, California 90024
J. DUGUNDJI*

Department of Mathematics

University of Southern California

Los Angeles, California 90007

D. Gilbarg and J. Milgram

Stanford University

Stanford, California 94305
University of Washington

Seattle, Washington 98105

ASSOCIATE EDITORS
E. F. BECKENBACH
B. H. NeumanN
F. WoLF
K. YosHIDA

\section{SUPPORTING INSTITUTIONS}

\author{
UNIVERSITY OF BRITISH COLUMBIA \\ CALIFORNIA INSTITUTE OF TECHNOLOGY \\ UNIVERSITY OF CALIFORNIA \\ MONTANA STATE UNIVERSITY \\ UNIVERSITY OF NEVADA \\ NEW MEXICO STATE UNIVERSITY \\ OREGON STATE UNIVERSITY \\ UNIVERSITY OF OREGON \\ OSAKA UNIVERSITY
}

\author{
UNIVERSITY OF SOUTHERN CALIFORNIA \\ STANFORD UNIVERSITY \\ UNIVERSITY OF TOKYO \\ UNIVERSITY OF UTAH \\ WASHINGTON STATE UNIVERSITY \\ UNIVERSITY OF WASHINGTON
* * * *
AMERICAN MATHEMATICAL SOCIETY \\ NAVAL WEAPONS CENTER
}

The Supporting Institutions listed above contribute to the cost of publication of this Journal, but they are not owners or publishers and have no responsibility for its content or policies.

Mathematical papers intended for publication in the Pacific Journal of Mathematics should be in typed form or offset-reproduced, (not dittoed), double spaced with large margins. Underline Greek letters in red, German in green, and script in blue. The first paragraph or two must be capable of being used separately as a synopsis of the entire paper. Items of the bibliography should not be cited there unless absolutely necessary, in which case they must be identified by author and Journal, rather than by item number. Manuscripts, in duplicate if possible, may be sent to any one of the four editors. Please classify according to the scheme of Math. Rev. Index to Vol. 39. All other communications to the editors should be addressed to the managing editor, or Elaine Barth, University of California, Los Angeles, California, 90024.

100 reprints are provided free for each article, only if page charges have been substantially paid. Additional copies may be obtained at cost in multiples of 50 .

The Pacific of Journal Mathematics is issued monthly as of January 1966. Regular subscription rate: $\$ 72.00$ a year (6 Vols., 12 issues). Special rate: $\$ 36.00$ a year to individual members of supporting institutions.

Subscriptions, orders for back numbers, and changes of address should be sent to Pacific Journal of Mathematics, 103 Highland Boulevard, Berkeley, California, 94708.

PUBLISHED BY PACIFIC JOURNAL OF MATHEMATICS, A NON-PROFIT CORPORATION

Printed at Kokusai Bunken Insatsusha (International Academic Printing Co., Ltd.), 270, 3-chome Totsuka-cho, Shinjuku-ku, Tokyo 160, Japan.

* C. R. DePrima California Institute of Technology, Pasadena, CA 91109, will replace J. Dugundji until August 1974.

Copyright (C) 1973 by Pacific Journal of Mathematics

Manufactured and first issued in Japan 


\section{Pacific Journal of Mathematics}

\section{Vol. 51, No. $2 \quad$ December, 1974}

Robert F. V. Anderson, Laplace transform methods in multivariate spectral theory .................................................. 339

William George Bade, Two properties of the Sorgenfrey plane . . . . . . . . . . . . 349

John Robert Baxter and Rafael Van Severen Chacon, Functionals on continuous

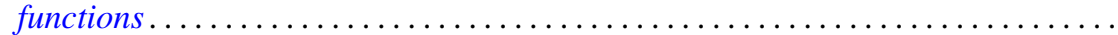

Phillip Wayne Bean, Helly and Radon-type theorems in interval convexity

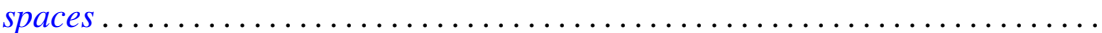

James Robert Boone, On k-quotient mappings $\ldots \ldots \ldots \ldots \ldots \ldots \ldots \ldots \ldots$

Ronald P. Brown, Extended prime spots and quadratic forms . . . . . . . . . . . .

William Hugh Cornish, Crawley's completion of a conditionally upper continuous lattice .............................................

Robert S. Cunningham, On finite left localizations ...................

Robert Jay Daverman, Approximating polyhedra in codimension one spheres

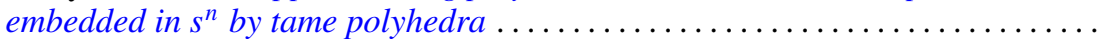

Burton I. Fein, Minimal splitting fields for group representations . . . . . . . . . . . .

Peter Fletcher and Robert Allen McCoy, Conditions under which a connected

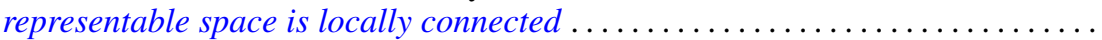

Jonathan Samuel Golan, Topologies on the torsion-theoretic spectrum of a noncommutative ring...

Manfred Gordon and Edward Martin Wilkinson, Determinants of Petrie matrices.

Alfred Peter Hallstrom, A counterexample to a conjecture on an integral condition for determining peak points (counterexample concerning peak points)........

E. R. Heal and Michael Windham, Finitely generated $F$-algebras with applications to Stein manifolds.

Denton Elwood Hewgill, On the eigenvalues of a second order elliptic operator in an unbounded domain ............................

Charles Royal Johnson, The Hadamard product of $A$ and $A^{*}$.

Darrell Conley Kent and Gary Douglas Richardson, Regular completions of Cauchy spaces.

Alan Greenwell Law and Ann L. McKerracher, Sharpened polynomial approximation

Bruce Stephen Lund, Subalgebras of finite codimension in the algebra of analytic functions on a Riemann surface. .

Robert Wilmer Miller, TTF classes and quasi-generators . .

Roberta Mura and Akbar H. Rhemtulla, Solvable groups in which every maximal partial order is isolated ....

Isaac Namioka, Separate continuity and joint continuity...

Alan Saleski, Entropy of self-homeomorphisms of statistical pseudo-metric

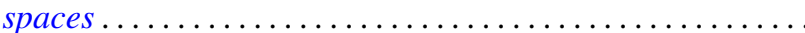

H. A. Seid, Cyclic multiplication operators on $L_{p}$-spaces .....

H. B. Skerry, On matrix maps of entire sequences ............

John Brendan Sullivan, A proof of the finite generation of invariants of a normal

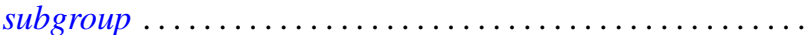

John Griggs Thompson, Nonsolvable finite groups all of whose local subgroups are

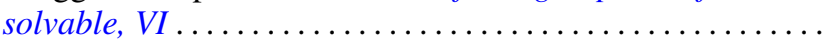

